\title{
MEMORY LOSS FOR TIME-DEPENDENT PIECEWISE EXPANDING SYSTEMS IN HIGHER DIMENSION
}

\author{
Chinmaya Gupta, William OtT and Andrei TöröK
}

\begin{abstract}
We prove a counterpart of exponential decay of correlations for certain nonstationary systems. Namely, given two probability measures absolutely continuous with respect to a reference measure, their quasi-Hölder distance (and in particular their $L^{1}$ distance) decreases exponentially under action by compositions of arbitrarily chosen maps close to those that are both piecewise expanding and mixing in a certain sense.
\end{abstract}

\section{Introduction}

This paper studies statistical properties of time-dependent dynamical systems. In such systems, the dynamical model itself is allowed to vary with time. An important example is the flow generated by a non-autonomous vector field. Perhaps the vector field depends on physical parameters that vary with time. We address memory loss for time-dependent dynamical systems, an analog of decay of correlations.

The memory loss problem has been studied extensively in the contexts of stochastic differential equations (SDEs), random dynamical systems ${ }^{1}$, and autonomous (timeindependent) deterministic dynamical systems. An SDE of the form

$$
d x_{t}=a\left(x_{t}\right) d t+\sum_{i=1}^{n} b_{i}\left(x_{t}\right) \circ d W_{t}^{i}
$$

gives rise to a stochastic flow of diffeomorphisms in which almost every Brownian path defines a time-dependent flow (see, e.g., [21]). Lyapunov exponents for such flows are known to be well-defined, non-random (they do not depend on the realization of the noise), and constant almost everywhere in phase space if the system is ergodic. Ergodic systems for which the greatest Lyapunov exponent $\lambda_{\max }$ is negative exhibit a phenomenon known as random sinks. Under suitable conditions, any ensemble of initial conditions will coalesce near a unique equilibrium point that evolves in time [23]. This phenomenon occurs in dissipative systems such as the Navier-Stokes system (see, e.g., $[29,30])$ and in certain coupled oscillator networks modelling neuronal activity [25]. Memory loss also occurs if $\lambda_{\max }>0$, for in this case initial distributions will track random SRB measures (see [24]) rather than random sinks. For further information about random dynamical systems, see, e.g., $[2,3]$.

Received by the editors November 30, 2012.

2010 Mathematics Subject Classification. 37C60, 37D20, 37D50, 82C99.

Key words and phrases. Hilbert metric, memory loss, mixing, piecewise expanding maps, timedependent dynamical systems.

${ }^{1}$ In the context of random dynamical systems, the maps are chosen according to a known distribution. 
We say that an autonomous deterministic system exhibits memory loss in the statistical sense if there exists a unique invariant measure $\nu$ that attracts absolutely continuous distributions $\rho_{0} \ll \nu$, that is $\rho_{t} \rightarrow \nu$ as $t \rightarrow \infty$ where $\rho_{t}$ denotes the dynamical evolution of $\rho_{0}$. Both the nature and speed of the convergence are of interest. Statistical memory loss and the closely related notion of decay of correlations have received a great deal of attention in this context (see, e.g., [11, 15-17, 26, 27, 31,32, 39, 45, 46]). Since time-dependent deterministic systems are out of equilibrium, we associate statistical memory loss not with initial distributions converging to invariant measures but rather with distances between pairs of initial distributions decreasing as the distributions evolve.

Important classes of time-dependent systems include dynamical systems with timevarying parameters and physical processes that take place in evolving environments. For example, consider a Lorentz gas (Sinai billiard) in which some of the scatterers move, perhaps due to bombardment by light particles. See [12] for an effort to model the movement of a heavy particle in this context. Stenlund et al. introduce a model of Sinai billiards with moving scatterers and prove an exponential memory loss result for this model [41]. Open systems (systems with holes) provide another important example, for perhaps the holes move over time. Mohapatra and Ott formulate a notion of conditional memory loss for time-dependent open systems and prove that this type of memory loss occurs at an exponential rate for a class of one-dimensional piecewisesmooth expanding maps with holes [33].

We do not assume any a priori knowledge of any statistical properties of the evolution of the dynamical model. Indeed, the stationarity of the process is irrelevant from our point of view. By contrast, knowledge of the statistical properties of the process typically plays a central role in random dynamical systems.

In this paper, we focus on time-dependent discrete-time systems: compositions of the form $f_{n} \circ f_{n-1} \circ \cdots \circ f_{1}$, where $\left(f_{i}\right)_{i}$ is a finite or infinite sequence of maps from a space $X$ into itself. Identify probability measures absolutely continuous with respect to the reference measure $\mu$ on $X$ with their their density functions (Radon-Nikodym derivatives with respect to $\mu$ ). We say that the system exhibits exponential memory loss in the statistical sense if given any initial densities $\varphi_{0}$ and $\psi_{0}$, the evolved densities $\varphi_{t}$ and $\psi_{t}$ satisfy $\left\|\varphi_{t}-\psi_{t}\right\|_{L^{1}(\mu)} \leqslant C_{\varphi_{0}, \psi_{0}} e^{-\Lambda t}$ for some $\Lambda>0$ independent of the initial measures.

We focus specifically on time-dependent piecewise $C^{1+\alpha}$ expanding systems in dimension at least two. Here the phase space $X$ is a Riemannian manifold and for each map $f_{i}$, there exists a finite or countably infinite collection $\left\{U_{i j}\right\}$ of pairwise disjoint open subsets of $X$ such that $\bigcup_{j} U_{i j}$ has full Riemannian volume for each $i$ and $f_{i} \mid U_{i j}$ is smooth for all relevant $i$ and $j$. The time-independent case (iterates of a single piecewise smooth expanding map) has received substantial attention, particularly with respect to the existence of absolutely continuous invariant probability measures (ACIPs) and exponential decay of correlations [1, 5, 7-10,13, 18, 20,34,38, 40, 42-44].

The ergodic theory of a time-independent piecewise smooth expanding system can be subtle in higher dimension because the domains on which the map is smooth can have complicated boundaries. This subtlety is magnified in the time-dependent case, wherein both the maps and the domains on which the maps are smooth can vary with time. 
In this paper, we prove that certain time-dependent piecewise $C^{1+\alpha}$ expanding systems in higher dimension exhibit exponential memory loss in the statistical sense. The current work builds on previous work covering time-dependent expanding systems and time-dependent piecewise expanding systems in one dimension $[22,37]$. This previous work uses the method of coupling, introduced in [46] and developed later in e.g., $[6,11,12]$. Here we use cones and the Hilbert projective metric (see, e.g., $[4,26,35,36])$. One could argue that coupling and the cone method are preferable to the spectral approach when dealing with time-dependent deterministic systems. For many random dynamical systems, one can study an averaged Perron-Frobenius operator (see, e.g., [14]); one cannot average in the time-dependent deterministic context.

\section{Statement of results}

We begin by defining a class of piecewise $C^{1+\alpha}$ expanding maps with good ergodic properties. The setup is based on that introduced by Saussol [40]. We describe perturbations of these maps and define the space of quasi-Hölder densities. Saussol proved that maps in this class admit finitely many ergodic ACIPs with quasi-Hölder densities. We state the local and global versions of our results at the end of this section; the global statement is a straightforward consequence of the local result.

Remark 2.1. Throughout this paper we use a fixed Hölder exponent $0<\alpha \leqslant$ Lip; unless otherwise stated, the maps are (piecewise) $C^{1+\alpha}$. For maps in $C^{1+\text { Lip }}$ we use $\alpha=1$ in the computations.

For $f: U \subset \mathbb{R}^{k} \rightarrow \mathbb{R}^{\ell}$ use as $C^{1+\alpha}$ and $C^{2}$ norms

$$
\|f\|_{C^{1+\alpha}}:=\|f\|_{C^{0}}+\|D f\|_{C^{\alpha}}, \quad\|f\|_{C^{2}}:=\|f\|_{C^{0}}+\|D f\|_{C^{0}}+\left\|D^{2} f\right\|_{C^{0}} .
$$

These extend in a straightforward way to the case when the domain or range is a subset of a compact manifold. ${ }^{2}$

Remark 2.2. For clarity of exposition we consider only maps on the $N$-dimensional torus. However, one can extend the proofs to arbitrary compact manifolds.

Let $\mathbb{T}^{N}=\mathbb{R}^{N} / \mathbb{Z}^{N}$ denote the $N$-dimensional torus and $\mu$ the normalized Lebesgue measure on $\mathbb{T}^{N}$. For a set $S \subset \mathbb{T}^{N}$ and $\varepsilon>0$, define

$$
B_{\varepsilon}(S):=\bigcup_{x \in S} B_{\varepsilon}(x)
$$

where $B_{\varepsilon}(x)$ or $B(x, \varepsilon)$ stand for the open ball of radius $\varepsilon$ centered at $x$.

2.1. Domains for piecewise continuous maps; the classes $\mathcal{R}(K), C(\mathcal{A})$ and $\boldsymbol{C}(\mathcal{R})$. Abusing notation, we refer to a family of open pairwise disjoint sets that covers $\mathbb{T}^{N}$ up to measure zero (e.g., $\mathcal{A} \in \mathcal{R}$ below) as an open partition. The maps we consider are piecewise continuous on such open partitions.

Definition 2.3 (partitions). Consider in $\mathbb{T}^{N}$ a finite family of pairwise disjoint open sets $\mathcal{A}:=\left\{U_{i}: 1 \leqslant i \leqslant M\right\}$.

We say that $\mathcal{A} \in \mathcal{R}(K)$ if it covers $\mathbb{T}^{N}$ up to measure zero and each $U_{i}$ has boundaries bounded piecewise in $C^{2}$ by $K$. More precisely:

\footnotetext{
${ }^{2}$ The particular formulas used for the norms in (2.1) are not important.
} 
(1) $\mu\left(\mathbb{T}^{N} \backslash \bigcup_{i} U_{i}\right)=0$;

(2) for each $i$ there are finitely many compact $C^{2}$ embedded codimension-one submanifolds $\left\{\Gamma_{i j}\right\}_{j}$ of $\mathbb{T}^{N}$ such that $\partial U_{i}$ is contained in the union $\bigcup_{j} \Gamma_{i j}$;

(3) the $C^{2}$ norm of each $\Gamma_{i j}$ is strictly less than $K$; that is, for each $\Gamma_{i j}$ there are finitely many $C^{2}$ charts $\Phi_{\ell ; i j}: B_{N} \subset \mathbb{R}^{N} \rightarrow W_{\ell ; i j} \subset \mathbb{T}^{N}$, where $B_{N}$ is the unit ball of $\mathbb{R}^{N}$, such that each $\Phi_{\ell ; i j}$ and $\Phi_{\ell ; i j}^{-1}$ have $C^{2}$-norm less than $K$ and $\Gamma_{i j} \subset \bigcup_{\ell} \Phi_{\ell ; i j}\left(B_{N} \cap\left(\mathbb{R}^{N-1} \oplus\{0\}\right)\right)$.

Denote by $\mathcal{R}$ the union $\bigcup_{K>0} \mathcal{R}(K)$.

For $\mathcal{A} \in \mathcal{R}$ we define, using the above notation

$$
\kappa(\mathcal{A}):=\sup _{x \in \mathbb{T}^{N}} \#\left\{\Gamma_{i j}: x \in \Gamma_{i j}\right\}
$$

which gives the maximum number of boundary components to which a point belongs.

Definition 2.4 (nearby partitions). We say that two open partitions $\mathcal{A}=\left\{U_{i}\right\}_{i}$ and $\widetilde{\mathcal{A}}=\left\{\widetilde{U}_{i}\right\}_{i}$ in $\mathcal{R}(K)$ are $\delta$-close if:

(1) the families $\mathcal{A}$ and $\widetilde{\mathcal{A}}$ have the same number of elements, and there is also a correspondence between the bounding submanifolds $\Gamma_{i j}$ and $\widetilde{\Gamma}_{i j}$;

(2) for each $i, \mathrm{~d}_{\text {Hausd }}\left(U_{i}, \widetilde{U}_{i}\right)<\delta$, where $\mathrm{d}_{\text {Hausd }}$ denotes Hausdorff distance; ${ }^{3}$

(3) for each $i, j$, the bounding submanifolds $\Gamma_{i j}$ and $\widetilde{\Gamma}_{i j}$ are less than $\delta$ apart in Hausdorff distance.

This defines a topology on $\mathcal{R}(K) .{ }^{4}$ It is not hard to see that

Lemma 2.5. Given $\mathcal{A} \in \mathcal{R}(K)$, there is $\delta>0$ such that $\kappa(\tilde{\mathcal{A}}) \leqslant \kappa(\mathcal{A})$ for each $\widetilde{\mathcal{A}} \in \mathcal{R}(K)$ that is $\delta$-close to $\mathcal{A}$.

Proof. Indeed, if $x_{n} \in \mathbb{T}^{N}$ is a point in $k$ boundary components of $\mathcal{A}_{n}$ with $\mathcal{A}_{n}$ being $\delta_{n}$-close to $\mathcal{A}, \delta_{n} \rightarrow 0$, then we can select a subsequence so that $x_{n} \rightarrow x$ and $x_{n} \in \cap_{(i, j) \in J} \Gamma_{i j}^{n}$ for a set $J$ with $k$ elements, with each boundary component $\Gamma_{i j}^{n}$ of $\mathcal{A}_{n}$ being $\delta_{n}$-close to the corresponding boundary component $\Gamma_{i j}$ of $\mathcal{A}$. As $n \rightarrow \infty$, this implies that $x \in \cap_{J} \Gamma_{i j}$.

Definition 2.6 (piecewise continuous maps). For $\mathcal{A} \in \mathcal{R}$, we write $f \in C(\mathcal{A})$ for functions $f: \mathbb{T}^{N} \rightarrow \mathbb{T}^{N}$ that are continuous on each $U \in \mathcal{A}$. Denote by $C(\mathcal{R})$ the union of all $C(\mathcal{A})$ with $\mathcal{A} \in \mathcal{R}$.

2.2. Piecewise expanding maps: the classes $\mathcal{M}$ and $\mathcal{M}^{*}$. We describe next the piecewise $C^{1+\alpha}$ expanding maps that we consider.

The main estimate we need, a Lasota-Yorke inequality derived by Saussol [40, Lemma 4.1], requires the properties described later in Definition 4.1; we consider a class $\mathcal{M}^{*}$ of maps satisfying those. We first introduce a class of piecewise expanding maps $\mathcal{M}$ that is easier to describe.

Notation 2.7. Denote by $\xi_{N}=\pi^{N / 2} /(N / 2)$ ! the volume of the unit ball in $\mathbb{R}^{N}$. $^{5}$

\footnotetext{
${ }^{3}$ Recall that $\mathrm{d}_{\text {Hausd }}(A, B):=\max \{\sup \{\operatorname{dist}(x, B) \mid x \in A\}, \sup \{\operatorname{dist}(y, A) \mid y \in B\}\}$.

${ }^{4}$ One can define a fundamental system of neighborhoods, as we do later in Section 2.3 for maps.

$5(N / 2)$ ! stands for $\Gamma(N / 2+1)$.
} 
Definition 2.8 (the class $\mathcal{M}$ ). For $0<s<1, K>0, \kappa>0$ such that (2.2) holds,

$$
s^{\alpha}+\left(\frac{4 s \kappa}{1-s}\right)\left(\frac{\xi_{N-1}}{\xi_{N}}\right)<1 \text {, }
$$

denote by $\mathcal{M}(s, K, \kappa)$ the piecewise $C^{1+\alpha}$ maps $f: \mathbb{T}^{N} \rightarrow \mathbb{T}^{N}$ that satisfy the following properties:

(1) $f \in C\left(\mathcal{A}_{1}\right)$ with $\mathcal{A}_{1}=\left\{U_{i}: 1 \leqslant i \leqslant M\right\} \in \mathcal{R}(K)$; we will refer to $\mathcal{A}_{1}$ by $\mathcal{A}_{1}(f)$;

(2) (backward contraction) for each $i,\left.f\right|_{U_{i}}$ is injective with a differentiable inverse and $\left\|D\left[\left(\left.f\right|_{U_{i}}\right)^{-1}\right]\right\|<s$

(3) for each $i$, both $\left.f\right|_{U_{i}}$ and all its partial derivatives extend to continuous functions on the closure of $U_{i}$;

(4) for each $i,\left\|\left.f\right|_{U_{i}}\right\|_{1+\alpha}<K$;

(5) $\kappa\left(\mathcal{A}_{1}\right) \leqslant \kappa$.

Definition 2.9 (the class $\left.\mathcal{M}^{*}\right)$. Denote by $\mathcal{M}^{*}\left(s, K, \kappa, \varepsilon_{0}\right)$ the set of maps $f \in$ $\mathcal{M}(s, K, \kappa)$ that can be extended to a neighborhood of the original sets $U_{i}$ as follows (we use the notations of Definition 2.8): for each $i$, there is an open set $V_{i} \supset \overline{U_{i}}$ and an extension $f_{(i)}: V_{i} \rightarrow \mathbb{T}^{N}$ of $\left.f\right|_{U_{i}}$ such that

(1) $f_{(i)}\left(V_{i}\right) \supset \overline{B_{\varepsilon_{0}}\left(f\left(U_{i}\right)\right)}$;

(2) $f_{(i)}$ is a $C^{1}$ diffeomorphism from $V_{i}$ to its image;

(3) (backward contraction of extensions) $\left\|D\left[f_{(i)}^{-1}\right]\right\|<s$ on $f_{(i)}\left(V_{i}\right)$;

(4) $\left\|f_{(i)}\right\|_{1+\alpha}<K$ on $V_{i}$.

Remark 2.10. For iterates of a single piecewise expanding map, not requiring a balance between complexity and expansion (as is condition (2.2)) can lead to maps with no ACIPs (see [9]).

2.3. Perturbations of maps in $\mathcal{M}$; the neighborhoods $\mathcal{N}(f, \delta ; s, K, \kappa)$. We describe next perturbations of $f \in \mathcal{M}$. This is similar to the topology used in [14, Section 2.4] and [19].

Definition 2.11 (nearby maps; $\mathcal{N}(\mathbf{f}, \boldsymbol{\delta} ; \mathbf{s}, \mathbf{K}, \boldsymbol{\kappa})$ ). Let $f, \tilde{f} \in \mathcal{M}(s, K, \kappa)$. We say that $\tilde{f}$ is a $\delta$-perturbation of $f$, denoted $\tilde{f} \in \mathcal{N}(f, \delta)=\mathcal{N}(f, \delta ; s, K, \kappa)$, if the following hold.

(1) $\tilde{f} \in C\left(\widetilde{\mathcal{A}}_{1}\right)$ with $\widetilde{\mathcal{A}}_{1} \in \mathcal{R}(K) \delta$-close to $\mathcal{A}_{1}(f)$, as described in Definition $2.4 ;^{6}$

(2) outside a $\delta$-neighborhood of the boundaries, ${ }^{7}$ the maps are $\delta$-close in $C^{1+\alpha}$ :

$$
\left\|\left.f\right|_{W_{i}}-\left.\tilde{f}\right|_{W_{i}}\right\|_{C^{1+\alpha}}<\delta \quad \text { for each } i
$$

where

$$
W_{i}:=\left\{x \in U_{i} \cap \widetilde{U}_{i} \mid \operatorname{dist}\left(x, U_{i}^{c}\right)>\delta, \quad \operatorname{dist}\left(x, \widetilde{U}_{i}^{c}\right)>\delta\right\} .
$$

\footnotetext{
${ }^{6}$ Note that one can represent an $f \in \mathcal{M}$ with more than one choice of $\mathcal{A}_{1}(f)$.

${ }^{7}$ By Definition 2.4, the boundaries of $\mathcal{A}_{1}$ and $\widetilde{\mathcal{A}}_{1}$ have Hausdorff distance at most $\delta$.
} 
It is not difficult to check that as $f$ and $\delta$ vary, the sets $\mathcal{N}(f, \delta ; s, K, \kappa)$ form a fundamental system of neighborhoods ${ }^{8}$, so they define a topology on $\mathcal{M}(s, K, \kappa)$.

2.4. Mixing maps: the class $\mathcal{E}$. Arbitrary compositions of piecewise $C^{1+\alpha}$ expanding maps in $\mathcal{M}^{*}$ do not necessarily exhibit exponential loss of memory. Indeed, a system defined by iterating a single piecewise $C^{1+\alpha}$ expanding map may not even be ergodic, and decay of correlations (memory loss) in this context requires mixing. We therefore formulate a type of mixing condition.

Definition 2.12 (the class $\mathcal{E}$ ). Let $\zeta_{1} \in(0,1)$ and $\zeta_{2} \in(1, \infty)$. We say a map $f: \mathbb{T}^{N} \rightarrow \mathbb{T}^{N}$ belongs to $\mathcal{E}\left(\zeta_{1}, \zeta_{2}\right)$ if for every finite partition $\mathcal{H}$ of $\mathbb{T}^{N}$ into hypercubes, there exists $J\left(\mathcal{H}, \zeta_{1}, \zeta_{2}\right)$ such that for every $H_{1}, H_{2} \in \mathcal{H}$, we have

$$
\zeta_{1}<\frac{\mu\left(H_{1} \cap f^{-i}\left(H_{2}\right)\right)}{\mu\left(H_{1}\right) \mu\left(H_{2}\right)}<\zeta_{2}
$$

for every $i \geqslant J\left(\mathcal{H}, \zeta_{1}, \zeta_{2}\right)$.

Remark 2.13. For fixed $\zeta_{1}, \zeta_{2}, \mathcal{H}$, and $i,(2.3)$ is an open condition with respect to the topology we have defined on $\mathcal{M}^{*}$. This is precisely how we use (2.3) in the proof of Theorem 2.16.

2.5. Densities: the set $\mathcal{D}$. We consider densities that are quasi-Hölder. These quasi-Hölder spaces were considered by Saussol [40], where more details can be found.

For $\varphi \in L^{1}(\mu)$ and a Borel set $S \subset \mathbb{T}^{N}$, define the oscillation of $\varphi$ on $S$ by

$$
\operatorname{osc}(\varphi, S):=\operatorname{Esup}(\varphi, S)-\operatorname{Einf}(\varphi, S) .
$$

Given $\varepsilon_{0}>0$, define the seminorm

$$
|\varphi|_{\alpha, \varepsilon_{0}}:=\sup _{0<\varepsilon \leqslant \varepsilon_{0}} \varepsilon^{-\alpha} \int_{\mathbb{T}^{N}} \operatorname{osc}\left(\varphi, B_{\varepsilon}(x)\right) d \mu(x) .
$$

The seminorms $|\varphi|_{\alpha, \varepsilon_{0}}$ are equivalent for different $\varepsilon_{0}$ 's. ${ }^{9}$ Define

$$
\mathrm{OSC}_{\alpha}:=\left\{\varphi \in L^{1}(\mu):|\varphi|_{\alpha, \varepsilon_{0}}<\infty\right\} .
$$

This space does not depend on $\varepsilon_{0}$, and contains the $\alpha$-Hölder functions. Define the norm $\|\cdot\|_{\alpha, \varepsilon_{0}}$ on $\mathrm{OSC}_{\alpha}$ by

$$
\|\varphi\|_{\alpha, \varepsilon_{0}}:=\|\varphi\|_{L^{1}(\mu)}+|\varphi|_{\alpha, \varepsilon_{0}} .
$$

Equipped with this norm, $\mathrm{OSC}_{\alpha}$ is a Banach space and the unit ball of $\mathrm{OSC}_{\alpha}$ is precompact in $L^{1}(\mu)$.

Our memory loss results hold for densities in the set

$$
\mathcal{D}:=\left\{\varphi \in \mathrm{OSC}_{\alpha}: \varphi \geqslant 0,\|\varphi\|_{L^{1}(\mu)}=1\right\} .
$$

Remark 2.14. Saussol [40] proves that maps somewhat more general ${ }^{10}$ than those in $\mathcal{M}^{*}$ admit an ACIP, whose density is in $\mathcal{D}$. This is obtained by proving that the Perron-Frobenius operator satisfies a Lasota-Yorke inequality on $\mathrm{OSC}_{\alpha}$, and therefore is quasi-compact.

\footnotetext{
${ }^{8}$ If $g \in \mathcal{N}\left(f_{1}, \delta_{1} ; s, K, \kappa\right) \cap \mathcal{N}\left(f_{2}, \delta_{2} ; s, K, \kappa\right)$ then $\mathcal{N}(g, \delta ; s, K, \kappa) \subset \mathcal{N}\left(f_{1}, \delta_{1} ; s, K, \kappa\right) \cap \mathcal{N}\left(f_{2}, \delta_{2} ;\right.$ $s, K, \kappa)$ for some $\delta>0$.

${ }^{9}$ For $0<\varepsilon_{1}<\varepsilon_{2}$, there are finitely many vectors $v_{i}$ such that $B_{\varepsilon_{2}}(x) \subset \cup_{i} B_{\varepsilon_{1}}\left(x+v_{i}\right)$ for all $x$.

${ }^{10}$ The main difference is that there can be countable many $U_{i}$ 's, and their boundaries need not be piecewise smooth. In that case, condition (2.2) is replaced by (PE5), described in Section 4.
} 
2.6. Results. As mentioned earlier, we need both sufficient expansion (for condition (2.2) to hold), and some form of mixing (of the type exhibited by maps in $\mathcal{E}$ ).

We formulate two types of results: local, governing arbitrary compositions of maps chosen from a small neighborhood of a fixed map in $\mathcal{E} \cap \mathcal{M}^{*}$, and global, wherein we move through a union of such neighborhoods; the latter is a simple consequence of the former.

We begin by setting some notation.

Notation 2.15. Given maps $f_{i}: \mathbb{T}^{N} \rightarrow \mathbb{T}^{N}$ for $i \in \mathbb{N}$, denote $F_{m, k}:=f_{m} \circ \cdots \circ f_{k}$ if $m \geqslant k$. Write $F_{m}$ for $F_{m, 1}$.

For a (non-singular) map $f: \mathbb{T}^{N} \rightarrow \mathbb{T}^{N}$, denote by $\mathcal{P}_{f}: L^{1}(\mu) \rightarrow L^{1}(\mu)$ the associated Perron-Frobenius operator:

$$
\int_{\mathbb{T}^{N}}(\varphi \circ f) \cdot \psi d \mu=\int_{\mathbb{T}^{N}} \varphi \cdot \mathcal{P}_{f}(\psi) d \mu \quad\left(\varphi \in L^{\infty}(\mu), \quad \psi \in L^{1}(\mu)\right) .
$$

In other words, the Perron-Frobenius map $\mathcal{P}_{f}$ describes the action of $f$ on ACIMs:

$$
f^{*}(\psi d \mu)=\mathcal{P}_{f}(\psi) d \mu .
$$

Recall that $\mathcal{P}_{f}$ does not increase the $L^{1}$-norm,

$$
\left\|\mathcal{P}_{f}(\psi)\right\|_{L^{1}} \leqslant\|\psi\|_{L^{1}}
$$

2.6.1. Local result. The main local theorem states that given two densities in $\mathcal{D}$, their distance with respect to $\|\cdot\|_{\alpha, \varepsilon_{0}}$ decreases at an exponential rate under action by arbitrary composition of maps that are close to a single map in $\mathcal{E} \cap \mathcal{M}^{*}$.

Theorem 2.16. Let $g \in \mathcal{E}\left(\zeta_{1}, \zeta_{2}\right) \cap \mathcal{M}^{*}\left(s, K, \kappa, \varepsilon_{0}\right)$. There exist $\Lambda<1$ and $\delta>0$ such that given $\varphi, \psi \in \mathcal{D}$, there exists $C_{\varphi, \psi}>0$ with the following property: for any sequence $\left(f_{i}\right)_{i=1}^{\infty}$ in $\mathcal{N}(g, \delta ; s, K, \kappa) \cap \mathcal{M}^{*}\left(s, K, \kappa, \varepsilon_{0}\right)$, we have

$$
\int_{\mathbb{T}^{N}}\left|\mathcal{P}_{F_{m}}(\varphi)-\mathcal{P}_{F_{m}}(\psi)\right| d \mu \leqslant\left\|\mathcal{P}_{F_{m}}(\varphi)-\mathcal{P}_{F_{m}}(\psi)\right\|_{\alpha, \varepsilon_{0}} \leqslant C_{\varphi, \psi} \Lambda^{m}
$$

for all $m \in \mathbb{N}$.

One can relax the hypotheses of the above theorem because, by the Whitney extension theorem, maps in $\mathcal{M}$ close to $f \in \mathcal{M}^{*}$ are in $\mathcal{M}^{*}$ provided the boundaries are suitable.

2.6.2. Global result. We note that many global formulations are possible. We give below such a result, but one can replace continuity of $\omega$ with weaker assumptions.

Let $a<b$ and let $\omega:[a, b] \rightarrow \mathcal{M}^{*}$ be a map. We discretize $\omega$ by considering sequences of the form $\left(\omega\left(t_{i}\right)\right)_{i}$ where $a \leqslant t_{1} \leqslant t_{2} \leqslant \cdots \leqslant b$. Let $\Omega_{m}:=\omega\left(t_{m}\right) \circ \cdots \circ \omega\left(t_{1}\right)$.

Theorem 2.17. Let $\omega:[a, b] \rightarrow \mathcal{M}^{*}\left(s, K, \kappa, \varepsilon_{0}\right)$ be a continuous map. Assume that

$$
\omega([a, b]) \subset \bigcup_{i=1}^{M} \mathcal{N}\left(g_{i}, \delta_{i} ; s, K, \kappa\right)
$$

where $g_{i} \in \mathcal{M}^{*}\left(s, K, \kappa, \varepsilon_{0}\right)$ are maps for which Theorem 2.16 holds with a corresponding $\delta_{i}>0$. 
Then there exists $\Lambda<1$ such that the following holds for any discretized sequence $\left(\omega\left(t_{i}\right)\right)_{i}$ : for every $\varphi, \psi \in \mathcal{D}$, there exists $C_{\varphi, \psi}^{\prime}>0$ such that

$$
\int_{\mathbb{T}^{N}}\left|\mathcal{P}_{\Omega_{m}}(\varphi)-\mathcal{P}_{\Omega_{m}}(\psi)\right| d \mu \leqslant\left\|\mathcal{P}_{\Omega_{m}}(\varphi)-\mathcal{P}_{\Omega_{m}}(\psi)\right\|_{\alpha, \varepsilon_{0}} \leqslant C_{\varphi, \psi}^{\prime} \Lambda^{m}
$$

for all relevant $m \in \mathbb{N}$.

\section{Proof of Theorem 2.16}

We use the theory of cones and a projective metric known as the Hilbert metric (see, e.g., [26, 28]). Saussol [40] uses this theory to obtain precise estimates on rates of correlation decay for maps in $\mathcal{M}^{*}$.

The proof proceeds as follows. We define a suitable cone $\mathcal{C}_{a} \subset \mathrm{OSC}_{\alpha}$. We then find a time $T \in \mathbb{N}$ such that $\mathcal{P}_{F_{i+T-1, i}}$ maps $\mathcal{C}_{a}$ strictly inside itself for all $i \in \mathbb{N}$. The diameter of $\mathcal{P}_{F_{i+T-1, i}}\left(\mathcal{C}_{a}\right)$ with respect to the Hilbert metric is bounded uniformly in $i$. The general theory of cones then implies that $\mathcal{P}_{F_{i+T-1, i}}$ is a contraction on $\mathcal{C}_{a}$ with a contraction factor that is uniform in $i$. Theorem 2.16 follows.

3.1. Invariance of a suitable convex cone. The following Lasota-Yorke inequality provides control of the oscillation seminorm of functions in $\mathrm{OSC}_{\alpha}$ under the action of the Perron-Frobenius operator. It is the crucial estimate that allows us to analyze the action of the Perron-Frobenius operator on $\mathcal{C}_{a}$. For completeness we provide the proof in Section 4.

Proposition 3.1 (Lasota-Yorke inequality [40]). Let $s$ and $\kappa$ be such that $(2.2)$ is satisfied, and $K, \varepsilon_{0}>0$. There are positive constants $\varepsilon_{L Y}=\varepsilon_{L Y}\left(s, K, \kappa, \varepsilon_{0}, N\right) \leqslant \varepsilon_{0}$, $\gamma_{L Y}=\gamma_{L Y}\left(s, K, \kappa, \varepsilon_{L Y}\right)<1$ and $K_{L Y}=K_{L Y}\left(s, K, \kappa, \varepsilon_{L Y}\right)$, such that:

$$
\text { if } f \in \mathcal{M}^{*}\left(s, K, \kappa, \varepsilon_{0}\right) \text { then } \mathcal{P}_{f} \text { maps } \mathrm{OSC}_{\alpha} \text { to itself and }
$$

$$
\left|\mathcal{P}_{f}(\varphi)\right|_{\alpha, \varepsilon_{L Y}} \leqslant \gamma_{L Y}|\varphi|_{\alpha, \varepsilon_{L Y}}+K_{L Y}\|\varphi\|_{L^{1}(\mu)} \quad \varphi \in \mathrm{OSC}_{\alpha}
$$

Proof. See Section 4, where we recall the proof of Saussol [40]. The constants $\varepsilon_{L Y}$, $\gamma_{L Y}$ and $K_{L Y}$ are described there.

Notation 3.2. We fix the above parameters $s, K, \kappa, \varepsilon_{0}$; the constants $\varepsilon_{L Y}, \gamma_{L Y}$ and $K_{L Y}$ are those given in Proposition 3.1.

We now define $\mathcal{C}_{a}$ and study the action of the Perron-Frobenius operator on it. The following parameters are used throughout the proof of Theorem 2.16.

(P1) $0<\sigma<1$

(P2) $\varepsilon_{\mathcal{H}}$ : choose such that $\varepsilon_{\mathcal{H}} \leqslant \varepsilon_{L Y}$. Let $\mathcal{H}$ be a partition of $\mathbb{T}^{N}$ into hypercubes such that

$$
\sup _{H \in \mathcal{H}} \operatorname{diam}(H) \leqslant \varepsilon_{\mathcal{H}} .
$$

(P3) $T \in \mathbb{N}$ : choose such that $T \geqslant J\left(\mathcal{H}, \zeta_{1}, \zeta_{2}\right)$. 
(P4) $a>0$ : the aperture of the cone $\mathcal{C}_{a}$. We choose the parameters $\varepsilon_{\mathcal{H}}, T$, and $a$ such that

$$
\begin{aligned}
\zeta_{1}-\zeta_{2} \varepsilon_{\mathcal{H}}^{\alpha} a & >0, \\
\left(\zeta_{1}-\zeta_{2} \varepsilon_{\mathcal{H}}^{\alpha} a\right)^{-1}\left(\gamma_{L Y}^{T} a+\frac{K_{L Y}}{1-\gamma_{L Y}}\right) & \leqslant \sigma a .
\end{aligned}
$$

The inequalities in (P4) may be simultaneously satisfied by first choosing $T$ sufficiently large, then $a$ sufficiently large, then $\varepsilon_{\mathcal{H}}$ sufficiently small, and finally increasing $T$ if necessary so that (P3) holds.

Once parameter selection is complete, let $\delta$ be sufficiently small so that (2.3) holds with $\zeta_{1}, \zeta_{2}$, and $\mathcal{H}$ fixed for every composition of $T$ maps chosen from $\mathcal{N}(g, \delta ; s, K, \kappa) \cap$ $\mathcal{M}^{*}\left(s, K, \kappa, \varepsilon_{0}\right)$.

Define

$$
\mathcal{C}_{a}=\left\{\varphi \in L^{1}(\mu): \varphi \neq 0, \varphi \geqslant 0,|\varphi|_{\alpha, \varepsilon_{L Y}} \leqslant a E[\varphi \mid \mathcal{H}]\right\}
$$

We now show that for every $i \in \mathbb{N}, \mathcal{P}_{F_{i+T-1, i}}$ maps $\mathcal{C}_{a}$ into $\mathcal{C}_{\sigma a}$. The following two lemmas accomplish this.

Lemma 3.3. For every $\varphi \in \mathcal{C}_{a}$ and $i \in \mathbb{N}$ we have

$$
\left(\zeta_{1}-\zeta_{2} \varepsilon_{\mathcal{H}}^{\alpha} a\right) \int_{\mathbb{T}^{N}} \varphi d \mu \leqslant E\left[\mathcal{P}_{F_{i+T-1, i}}(\varphi) \mid \mathcal{H}\right] \leqslant \zeta_{2}\left(1+a \varepsilon_{\mathcal{H}}^{\alpha}\right) \int_{\mathbb{T}^{N}} \varphi d \mu
$$

Proof of Lemma 3.3. Write $F=F_{i+T-1, i}$. For $x \in \mathbb{T}^{N}$, let $H(x)$ denote the element of $\mathcal{H}$ that contains $x$. We have

$$
\begin{aligned}
E\left[\mathcal{P}_{F}(\varphi) \mid \mathcal{H}\right](x) & =\frac{1}{\mu(H(x))} \int_{H(x)} \mathcal{P}_{F}(\varphi) d \mu \\
& =\frac{1}{\mu(H(x))} \int_{F^{-1}(H(x))} \varphi d \mu \\
& =\frac{1}{\mu(H(x))} \sum_{H^{\prime} \in \mathcal{H}} \int_{H^{\prime} \cap F^{-1}(H(x))} \varphi(z) d \mu(z) .
\end{aligned}
$$

Bounding $\varphi$ from below, for $\mu$ almost every $z$ in $H^{\prime} \cap F^{-1}(H(x))$ we have

$$
\begin{aligned}
\varphi(z) & \geqslant\left(\frac{1}{\mu\left(H^{\prime}\right)} \int_{H^{\prime}} \varphi d \mu\right)-\operatorname{osc}\left(\varphi, H^{\prime}\right) \\
& \geqslant \frac{1}{\mu\left(H^{\prime}\right)}\left(\int_{H^{\prime}} \varphi d \mu-\int_{H^{\prime}} \operatorname{osc}\left(\varphi, B\left(y, \varepsilon_{\mathcal{H}}\right)\right) d y\right) .
\end{aligned}
$$


Integrating gives

$$
\begin{aligned}
E\left[\mathcal{P}_{F}(\varphi) \mid \mathcal{H}\right](x) \geqslant & \sum_{H^{\prime} \in \mathcal{H}} \frac{1}{\mu(H(x))} \int_{H^{\prime} \cap F^{-1}(H(x))} \frac{1}{\mu\left(H^{\prime}\right)} \\
& \times\left(\int_{H^{\prime}} \varphi d \mu-\int_{H^{\prime}} \operatorname{osc}\left(\varphi, B\left(y, \varepsilon_{\mathcal{H}}\right)\right) d y\right) d \mu(z) \\
= & \sum_{H^{\prime} \in \mathcal{H}} \frac{\mu\left(H^{\prime} \cap F^{-1}(H(x))\right)}{\mu(H(x)) \mu\left(H^{\prime}\right)}\left(\int_{H^{\prime}} \varphi d \mu-\int_{H^{\prime}} \operatorname{osc}\left(\varphi, B\left(y, \varepsilon_{\mathcal{H}}\right)\right) d y\right) \\
\geqslant & \zeta_{1} \int_{\mathbb{T}^{N}} \varphi d \mu-\zeta_{2}|\varphi|_{\alpha, \varepsilon_{L} Y} \varepsilon_{\mathcal{H}}^{\alpha} \\
\geqslant & \left(\zeta_{1}-\zeta_{2} \varepsilon_{\mathcal{H}}^{\alpha} a\right) \int_{\mathbb{T}^{N}} \varphi d \mu .
\end{aligned}
$$

The upper bound

$$
E\left[\mathcal{P}_{F}(\varphi) \mid \mathcal{H}\right](x) \leqslant \zeta_{2}\left(1+a \varepsilon_{\mathcal{H}}^{\alpha}\right) \int_{\mathbb{T}^{N}} \varphi d \mu
$$

is established in a similar fashion.

Lemma 3.4. For every $i \in \mathbb{N}$ we have

$$
\mathcal{P}_{F_{i+T-1, i}}\left(\mathcal{C}_{a}(\mathcal{H})\right) \subset \mathcal{C}_{\sigma a}(\mathcal{H}) .
$$

Proof of Lemma 3.4. Write $F=F_{i+T-1, i}$. Iterating (3.1) and using (3.2), we have

$$
\begin{aligned}
\left|\mathcal{P}_{F}(\varphi)\right|_{\alpha, \varepsilon_{L Y}} & \leqslant \gamma_{L Y}^{T}|\varphi|_{\alpha, \varepsilon_{L Y}}+\frac{K_{L Y}}{1-\gamma_{L Y}}\|\varphi\|_{L^{1}(\mu)} \\
& \leqslant\|\varphi\|_{L^{1}(\mu)}\left(\gamma_{L Y}^{T} a+\frac{K_{L Y}}{1-\gamma_{L Y}}\right) \\
& \leqslant\left(\zeta_{1}-\zeta_{2} \varepsilon_{\mathcal{H}}^{\alpha} a\right)^{-1}\left(\gamma_{L Y}^{T} a+\frac{K_{L Y}}{1-\gamma_{L Y}}\right) E\left[\mathcal{P}_{F}(\varphi) \mid \mathcal{H}\right] \\
& \leqslant \sigma a E\left[\mathcal{P}_{F}(\varphi) \mid \mathcal{H}\right] .
\end{aligned}
$$

3.2. $\mathcal{P}_{\boldsymbol{F}_{i+T-1, i}}$ is a contraction on $\mathcal{C}_{\boldsymbol{a}}$. See, e.g., $[4,26,35,36]$ for information on cones and the Hilbert projective metric. Here we briefly introduce what we need in our context.

Define the partial order $\prec$ on $\mathcal{C}_{a}$ by declaring that $\varphi \prec \psi$ if $\psi-\varphi \in \mathcal{C}_{a}$. The Hilbert metric $\Theta$ is defined on $\mathcal{C}_{a}$ by

$$
\Theta(\varphi, \psi)=\log \left(\frac{\inf \{s>0: \psi \prec s \varphi\}}{\sup \{r>0: r \varphi \prec \psi\}}\right) .
$$

Theorem $3.5([4])$. Let $i \in \mathbb{N}$. Define

$$
\Delta_{i}=\sup _{\varphi^{*}, \psi^{*} \in \mathcal{P}_{F_{i+T-1, i}}\left(\mathcal{C}_{a}\right)} \Theta\left(\varphi^{*}, \psi^{*}\right) .
$$

For every $\varphi, \psi \in \mathcal{C}_{a}$, we have

$$
\Theta\left(\mathcal{P}_{F_{i+T-1, i}}(\varphi), \mathcal{P}_{F_{i+T-1, i}}(\psi)\right) \leqslant \tanh \left(\frac{\Delta_{i}}{4}\right) \Theta(\varphi, \psi) .
$$


Here $\tanh (\infty)=1$.

The following lemma provides an upper bound on the diameter of $\mathcal{P}_{F_{i+T-1, i}}\left(\mathcal{C}_{a}\right)$ in $\mathcal{C}_{a}$ that is uniform in $i$.

Lemma 3.6. For every $i \in \mathbb{N}$ and all $\varphi, \psi \in \mathcal{C}_{a}$, we have

$$
\Theta\left(\mathcal{P}_{F_{i+T-1, i}}(\varphi), \mathcal{P}_{F_{i+T-1, i}}(\psi)\right) \leqslant \Delta:=2 \log \left(\frac{1+\sigma}{1-\sigma}\right)+2 \log \left(\frac{\zeta_{2}\left(1+a \varepsilon_{\mathcal{H}}^{\alpha}\right)}{\zeta_{1}-\zeta_{2} \varepsilon_{\mathcal{H}}^{\alpha} a}\right)
$$

Proof of Lemma 3.6. Let $\varphi^{*}, \psi^{*} \in \mathcal{C}_{\sigma a}$. Let $r$ and $s$ be such that

$$
r \varphi^{*} \prec \psi^{*} \prec s \varphi^{*} \text {. }
$$

Looking at $\psi^{*}-r \varphi^{*}$, we have

$$
\begin{aligned}
\left|\psi^{*}-r \varphi^{*}\right|_{\alpha, \varepsilon_{L Y}} & \leqslant\left|\psi^{*}\right|_{\alpha, \varepsilon_{L Y}}+r\left|\varphi^{*}\right|_{\alpha, \varepsilon_{L Y}} \\
& \leqslant \sigma a E\left[\psi^{*} \mid \mathcal{H}\right]+\operatorname{roaE}\left[\varphi^{*} \mid \mathcal{H}\right] .
\end{aligned}
$$

Therefore, $\psi^{*}-r \varphi^{*} \in \mathcal{C}_{a}$ if

$$
\sigma a E\left[\psi^{*} \mid \mathcal{H}\right]+\operatorname{r\sigma aE}\left[\varphi^{*} \mid \mathcal{H}\right] \leqslant a E\left[\psi^{*}-r \varphi^{*} \mid \mathcal{H}\right],
$$

or equivalently,

$$
r \leqslant\left(\frac{1-\sigma}{1+\sigma}\right) \frac{E\left[\psi^{*} \mid \mathcal{H}\right]}{E\left[\varphi^{*} \mid \mathcal{H}\right]}
$$

Arguing analogously, $s \varphi^{*}-\psi^{*} \in \mathcal{C}_{a}$ if

$$
\left(\frac{1+\sigma}{1-\sigma}\right) \frac{E\left[\psi^{*} \mid \mathcal{H}\right]}{E\left[\varphi^{*} \mid \mathcal{H}\right]} \leqslant s .
$$

Bounds (3.4) and (3.5) imply

$$
\begin{aligned}
\Theta\left(\varphi^{*}, \psi^{*}\right) & \leqslant \log \left(\left(\frac{1+\sigma}{1-\sigma}\right) \operatorname{Esup}\left(\frac{E\left[\psi^{*} \mid \mathcal{H}\right]}{E\left[\varphi^{*} \mid \mathcal{H}\right]}\right)\right)-\log \left(\left(\frac{1-\sigma}{1+\sigma}\right) \operatorname{Einf}\left(\frac{E\left[\psi^{*} \mid \mathcal{H}\right]}{E\left[\varphi^{*} \mid \mathcal{H}\right]}\right)\right) \\
& =2 \log \left(\frac{1+\sigma}{1-\sigma}\right)+\log \left(\left\|\frac{E\left[\psi^{*} \mid \mathcal{H}\right]}{E\left[\varphi^{*} \mid \mathcal{H}\right]}\right\|_{L^{\infty}}\left\|\frac{E\left[\varphi^{*} \mid \mathcal{H}\right]}{E\left[\psi^{*} \mid \mathcal{H}\right]}\right\|_{L^{\infty}}\right) .
\end{aligned}
$$

Now let $\varphi, \psi \in \mathcal{C}_{a}$. Write $F_{i+T-1, i}=F$. Using Lemma 3.4, estimate (3.6), and Lemma 3.3, we have

$$
\Theta\left(\mathcal{P}_{F}(\varphi), \mathcal{P}_{F}(\psi)\right) \leqslant \Delta:=2 \log \left(\frac{1+\sigma}{1-\sigma}\right)+2 \log \left(\frac{\zeta_{2}\left(1+a \varepsilon_{\mathcal{H}}^{\alpha}\right)}{\zeta_{1}-\zeta_{2} \varepsilon_{\mathcal{H}}^{\alpha} a}\right)
$$

Since the diameter of $\mathcal{P}_{F_{i+T-1, i}}\left(\mathcal{C}_{a}\right)$ is finite, Theorem 3.5 implies that $\mathcal{P}_{F_{i+T-1, i}}$ is a contraction on $\mathcal{C}_{a}$.

Proposition 3.7. For every $i \in \mathbb{N}$ and all $\varphi, \psi \in \mathcal{C}_{a}$, we have

$$
\Theta\left(\mathcal{P}_{F_{i+T-1, i}}(\varphi), \mathcal{P}_{F_{i+T-1, i}}(\psi)\right) \leqslant \tanh \left(\frac{\Delta}{4}\right) \Theta(\varphi, \psi) .
$$




\subsection{Comparing the $\mathrm{OSC}_{\alpha}$ distance to the $\Theta$ distance.}

Lemma 3.8. For every $m \in \mathbb{N}$ and all $\varphi, \psi \in \mathcal{D} \cap \mathcal{C}_{a}$, we have

$$
\left\|\mathcal{P}_{F_{m T}}(\psi)-\mathcal{P}_{F_{m T}}(\varphi)\right\|_{\alpha, \varepsilon_{L Y}} \leqslant\left(a\left(1+\varepsilon_{\mathcal{H}}^{\alpha}\right)+(2+a)\right) \Theta\left(\mathcal{P}_{F_{m T}}(\psi), \mathcal{P}_{F_{m T}}(\varphi)\right) .
$$

Proof of Lemma 3.8. Write $F=F_{m T}$. Suppose $r, s \geqslant 0$ are such that $r \leqslant 1 \leqslant s$ and

$$
r \mathcal{P}_{F}(\varphi) \prec \mathcal{P}_{F}(\psi) \prec s \mathcal{P}_{F}(\varphi) .
$$

Estimating the $L^{1}$ norm of $\mathcal{P}_{F}(\psi)-\mathcal{P}_{F}(\varphi)$, we have

$$
\begin{aligned}
\left\|\mathcal{P}_{F}(\psi)-\mathcal{P}_{F}(\varphi)\right\|_{L^{1}(\mu)}= & \int_{\mathbb{T}^{N}}\left|\mathcal{P}_{F}(\psi)-(r+(1-r)) \mathcal{P}_{F}(\varphi)\right| d \mu \\
\leqslant & \int_{\mathbb{T}^{N}}\left|\mathcal{P}_{F}(\psi)-r \mathcal{P}_{F}(\varphi)\right| d \mu+(1-r) \\
\leqslant & \left|\mathcal{P}_{F}(\psi)-r \mathcal{P}_{F}(\varphi)\right|_{\alpha, \varepsilon_{L Y}} \varepsilon_{\mathcal{H}}^{\alpha} \\
& +\int_{\mathbb{T}^{N}} \mathcal{P}_{F}(\psi)-r \mathcal{P}_{F}(\varphi) d \mu+(1-r) \\
= & \left|\mathcal{P}_{F}(\psi)-r \mathcal{P}_{F}(\varphi)\right|_{\alpha, \varepsilon_{L Y}} \varepsilon_{\mathcal{H}}^{\alpha}+2(1-r) .
\end{aligned}
$$

Estimating the oscillation seminorm of $\mathcal{P}_{F}(\psi)-\mathcal{P}_{F}(\varphi)$, we have

$$
\left|\mathcal{P}_{F}(\psi)-\mathcal{P}_{F}(\varphi)\right|_{\alpha, \varepsilon_{L Y}} \leqslant\left|\mathcal{P}_{F}(\psi)-r \mathcal{P}_{F}(\varphi)\right|_{\alpha, \varepsilon_{L Y}}+(1-r)\left|\mathcal{P}_{F}(\varphi)\right|_{\alpha, \varepsilon_{L} Y} .
$$

Estimates (3.9) and (3.10) imply

$\left\|\mathcal{P}_{F}(\psi)-\mathcal{P}_{F}(\varphi)\right\|_{\alpha, \varepsilon_{L Y}} \leqslant a\left(1+\varepsilon_{\mathcal{H}}^{\alpha}\right) E\left[\mathcal{P}_{F}(\psi)-r \mathcal{P}_{F}(\varphi) \mid \mathcal{H}\right]+(1-r)\left(2+a E\left[\mathcal{P}_{F}(\varphi) \mid \mathcal{H}\right]\right)$.

Integrating gives

$$
\left\|\mathcal{P}_{F}(\psi)-\mathcal{P}_{F}(\varphi)\right\|_{\alpha, \varepsilon_{L Y}} \leqslant(1-r)\left(a\left(1+\varepsilon_{\mathcal{H}}^{\alpha}\right)+(2+a)\right) .
$$

Finally, since $1-r \leqslant-\log (r) \leqslant \log (s / r)$ for $s \geqslant 1$, we conclude that

$$
\left\|\mathcal{P}_{F}(\psi)-\mathcal{P}_{F}(\varphi)\right\|_{\alpha, \varepsilon_{L Y}} \leqslant\left(a\left(1+\varepsilon_{\mathcal{H}}^{\alpha}\right)+(2+a)\right) \Theta\left(\mathcal{P}_{F}(\psi), \mathcal{P}_{F}(\varphi)\right) .
$$

3.4. Completion of the proof of Theorem 2.16. Let $\varphi, \psi \in \mathcal{D} \cap \mathcal{C}_{a}$. Let $m \in \mathbb{N}$. Write $m=k T+i$, where $k \in \mathbb{Z}^{+}$and $0 \leqslant i<T$. Using (3.1), Lemma 3.8, and Proposition 3.7, we have

$$
\begin{aligned}
&\left\|\mathcal{P}_{F_{m}}(\varphi)-\mathcal{P}_{F_{m}}(\psi)\right\|_{\alpha, \varepsilon_{L Y}} \\
& \leqslant\left(1+\frac{K_{L Y}}{1-\gamma_{L Y}}\right)\left\|\mathcal{P}_{F_{k T}}(\varphi)-\mathcal{P}_{F_{k T}}(\psi)\right\|_{\alpha, \varepsilon_{L Y}} \\
& \leqslant\left(1+\frac{K_{L Y}}{1-\gamma_{L Y}}\right)\left(a\left(1+\varepsilon_{\mathcal{H}}^{\alpha}\right)+(2+a)\right) \Theta\left(\mathcal{P}_{F_{k T}}(\varphi), \mathcal{P}_{F_{k T}}(\psi)\right) \\
& \leqslant\left(1+\frac{K_{L Y}}{1-\gamma_{L Y}}\right)\left(a\left(1+\varepsilon_{\mathcal{H}}^{\alpha}\right)+(2+a)\right) \\
& \quad \times \max \{\Delta, 1\} \cdot \tanh ^{-2}\left(\frac{\Delta}{4}\right)\left(\tanh \left(\frac{\Delta}{4}\right)^{1 / T}\right)^{m}
\end{aligned}
$$


For general $\varphi, \psi \in \mathcal{D}$, choose $\eta_{\varphi, \psi}>0$ sufficiently large so that

$$
\frac{\varphi+\eta_{\varphi, \psi}}{1+\eta_{\varphi, \psi}} \in \mathcal{D} \cap \mathcal{C}_{a}, \quad \frac{\psi+\eta_{\varphi, \psi}}{1+\eta_{\varphi, \psi}} \in \mathcal{D} \cap \mathcal{C}_{a}
$$

We have established (2.6) with

$$
\begin{aligned}
C_{\varphi, \psi}= & K_{\varepsilon_{L Y}, \varepsilon_{0}}\left(1+\eta_{\varphi, \psi}\right)\left(1+\frac{K_{L Y}}{1-\gamma_{L Y}}\right) \\
& \times\left(a\left(1+\varepsilon_{\mathcal{H}}^{\alpha}\right)+(2+a)\right) \cdot \max \{\Delta, 1\} \cdot \tanh ^{-2}\left(\frac{\Delta}{4}\right) \\
\Lambda= & \tanh \left(\frac{\Delta}{4}\right)^{1 / T} .
\end{aligned}
$$

Here the constant $K_{\varepsilon_{L Y}, \varepsilon_{0}}$ accounts for the equivalence of $\|\cdot\|_{\alpha, \varepsilon_{L Y}}$ and $\|\cdot\|_{\alpha, \varepsilon_{0}}$.

\section{Proof of Proposition 3.1}

This result is in Saussol [40], for a class of piecewise expanding maps in which (PE5) below replaces (2.2). We are repeating the proof here to clarify how the constants are determined.

For reference, in Definition 4.1 we describe (using partially his notation) maps considered by Saussol [40, Section 2]. In Saussol's case the family $\left\{U_{i}\right\}_{i}$ can be countable and the domain of $f$ is a compact set $\Omega \subset \mathbb{R}^{N}$ that is equal to the closure of its interior. We rewrote the properties for maps on $\mathbb{T}^{N}$.

Definition 4.1 (Saussol [40]). We say that $f: \mathbb{T}^{N} \rightarrow \mathbb{T}^{N}$ is an admissible piecewise expanding map if there exists a finite collection $\mathcal{A}_{1}=\mathcal{A}_{1}(f)=\left\{U_{i}: 1 \leqslant i \leqslant M\right\}$ of pairwise disjoint open sets, $0<\varepsilon_{*}<1 / 2, s<1, c_{\operatorname{detH}}>0$ such that the following hold.

(PE1) (extension) For every $1 \leqslant i \leqslant M$ there exists an open set $V_{i}$ satisfying $V_{i} \supset \bar{U}_{i}$ such that

(a) $f \mid U_{i}$ extends to a map $f_{(i)}: V_{i} \rightarrow \mathbb{T}^{N}$

(b) $f_{(i)}\left(V_{i}\right) \supset \overline{B_{\varepsilon_{*}}\left(f\left(U_{i}\right)\right)}$.

(PE2) (regularity)

(a) the map $f_{(i)}$ is a $C^{1}$ diffeomorphism from $V_{i}$ to $f_{(i)}\left(V_{i}\right)$ and

(b) the determinant of $D\left[f_{(i)}^{-1}\right]$ is uniformly $\alpha$-Hölder in the following sense: ${ }^{11}$ for $0<\varepsilon \leqslant s \varepsilon_{*}, z \in V_{i}$ and $x \in B_{\varepsilon}(z) \cap V_{i}$,

$$
\left|\frac{1}{\operatorname{det} D_{x} f_{(i)}}-\frac{1}{\left.\operatorname{det} D_{z} f_{(i)}\right]}\right| \leqslant c_{\operatorname{detH}} \frac{\varepsilon^{\alpha}}{\left|\operatorname{det} D_{z} f_{(i)}\right|}
$$

(PE3) $\mu\left(\mathbb{T}^{N} \backslash \bigcup_{i=1}^{M} U_{i}\right)=0$.

\footnotetext{
${ }^{11}$ Saussol has this stated for $\left|\operatorname{det} D_{u}\left[f_{(i)}^{-1}\right]-\operatorname{det} D_{v}\left[f_{(i)}^{-1}\right]\right|$ with $w \in f_{(i)}\left(V_{i}\right), u, v \in B_{\varepsilon}(w) \cap$ $f_{(i)}\left(V_{i}\right)$.
} 
(PE4) (backward contraction) For all $1 \leqslant i \leqslant M$ and $x, y \in f_{(i)}\left(V_{i}\right)$, we have $\operatorname{dist}_{\mathbb{T}^{N}}(x, y) \leqslant \varepsilon_{*} \Longrightarrow \operatorname{dist}_{\mathbb{T}^{N}}\left(\left(f_{(i)}\right)^{-1}(x),\left(f_{(i)}\right)^{-1}(y)\right)<s \cdot \operatorname{dist}_{\mathbb{T}^{N}}(x, y)$, where dist $_{\mathbb{T}^{N}}$ is the standard metric on $\mathbb{T}^{N}$.

(PE5) (cutting versus expansion) For $0<\varepsilon \leqslant \varepsilon_{*}$ introduce the following constants and assume $\eta\left(f, \varepsilon_{*}\right)$ is finite ${ }^{12}$

$$
\begin{aligned}
& \rho\left(f, \varepsilon, \varepsilon_{*}\right):=\sup _{x \in \mathbb{T}^{N}} \sum_{i=1}^{M} \frac{\mu\left(\left(f_{(i)}\right)^{-1}\left(B_{\varepsilon}\left(\partial f\left(U_{i}\right)\right)\right) \cap B_{(1-s) \varepsilon_{*}}(x)\right)}{\mu\left(B_{(1-s) \varepsilon_{*}}(x)\right)}, \\
& \eta\left(f, \varepsilon_{*}\right):=s^{\alpha}+2 \sup _{\varepsilon \leqslant \varepsilon_{*}} \frac{\rho\left(f, \varepsilon, \varepsilon_{*}\right)}{\varepsilon^{\alpha}} \varepsilon_{*}{ }^{\alpha} .
\end{aligned}
$$

Following Saussol, we derive in Section 4.3 the following Lasota-Yorke inequality: Theorem 4.2 (Saussol [40], Lemma 4.1). Assume $f$ satisfies (PE1)-(PE5). Then

$$
\left|\mathcal{P}_{f}(\varphi)\right|_{\alpha, \varepsilon_{*}} \leqslant \gamma_{S}|\varphi|_{\alpha, \varepsilon_{*}}+K_{S}\|\varphi\|_{L^{1}(\mu)},
$$

where

$$
\begin{aligned}
\gamma_{S} & =\left(1+c_{\operatorname{detH}} s^{\alpha} \varepsilon_{*}{ }^{\alpha}\right) \eta\left(f, \varepsilon_{*}\right), \\
K_{S} & =2 c_{\operatorname{detH}} s^{\alpha}+2\left(1+c_{\operatorname{detH}} s^{\alpha} \varepsilon_{*}{ }^{\alpha}\right)\left(\sup _{\varepsilon \leqslant \varepsilon_{*}} \frac{\rho\left(f, \varepsilon, \varepsilon_{*}\right)}{\varepsilon^{\alpha}}\right) .
\end{aligned}
$$

Proof. See Section 4.3.

4.1. Proof of Proposition 3.1. We verify that maps in $\mathcal{M}^{*}\left(s, K, \kappa, \varepsilon_{0}\right)$ satisfy the properties (PE1)-(PE5) and estimate the constants. Let $f \in \mathcal{M}^{*}\left(s, K, \kappa, \varepsilon_{0}\right)$. For $\varepsilon_{L Y} \leqslant \varepsilon_{0}$, the properties that are not clear are (b) in (PE2) and (PE5).

Condition (PE2)(b): Since $\left\|D_{y} f_{(i)}\right\| \leqslant K$ for $y \in V_{i}$ and $\left\|D f_{(i)}\right\|_{C^{\alpha}\left(V_{i}\right)} \leqslant K$, it follows that for $x, z \in V_{i}$,

$$
\begin{aligned}
\left|\left(\operatorname{det} D_{x} f_{(i)}\right)^{-1}-\left(\operatorname{det} D_{z} f_{(i)}\right)^{-1}\right|= & \left|\operatorname{det} D_{x} f_{(i)}-\operatorname{det} D_{z} f_{(i)}\right| \\
& \times\left|\operatorname{det} D_{x} f_{(i)}\right|^{-1}\left|\operatorname{det} D_{z} f_{(i)}\right|^{-1} \\
\leqslant & C_{\operatorname{det}}(N, K, s)\left\|D f_{(i)}\right\|_{C^{\alpha}} \\
& \times\left|\operatorname{det} D_{z} f_{(i)}\right|^{-1} \operatorname{dist}_{\mathbb{T}^{N}}(x, z)^{\alpha}
\end{aligned}
$$

where

$$
\begin{aligned}
C_{\operatorname{det}}(N, K, s)= & \sup \left\{\frac{|\operatorname{det} A-\operatorname{det} B|}{\|A-B\|} \mid A, B \in \operatorname{Mat}_{N \times N}(\mathbb{R}), A \neq B,\|A\|,\|B\| \leqslant K\right\} \\
& \times \sup \left\{\left|\operatorname{det}\left(A^{-1}\right)\right| \mid A \in \operatorname{GL}(N, \mathbb{R}),\left\|A^{-1}\right\| \leqslant s<1\right\}<\infty .
\end{aligned}
$$

In conclusion,

$$
c_{\text {detH }} \leqslant C_{\text {det }}(N, K, s) K .
$$

Condition (PE5): Saussol [40, Lemma 2.1] shows that (2.2) implies $\eta_{\varepsilon_{*}}(f)<1$ if $\varepsilon_{*}$ is chosen sufficiently small. The key estimate are equations (3) and (4) in the proof of Saussol's Lemma 2.1. In Lemma 4.3, proven below, we repeat this argument with a more precise statement.

\footnotetext{
${ }^{12}$ Saussol requires in addition that $\eta_{\varepsilon_{*}}(f):=\sup _{\gamma \leqslant \varepsilon_{*}} \eta(f, \gamma)<1$.
} 
Lemma 4.3. Let $\Gamma \subset \mathbb{T}^{N}$ be a compact codimension-one embedded submanifold with $C^{2}$-norm bounded by $K$. Then

$$
\sup _{x} \mu\left(B_{\nu}(\Gamma) \cap B_{\omega}(x)\right)=2 \nu \omega^{N-1} \xi_{N-1}\left(1+o_{K}(1)\right) \text { as } \max \{\nu, \omega\} \rightarrow 0^{+}
$$

where $o_{K}(1)$ means that this asymptotic is determined only by the value $K$.

To estimate $\rho(f, \varepsilon, \gamma)$, because of the backward contraction, we need Lemma 4.3 with $\nu=s \varepsilon<\gamma$ and $\omega=(1-s) \gamma$, where $0<\varepsilon \leqslant \gamma \leqslant \varepsilon_{0}$ :

$$
\rho(f, \varepsilon, \gamma) \leqslant \kappa \frac{2(s \varepsilon)[(1-s) \gamma]^{N-1} \xi_{N-1}\left(1+o_{K}(1)\right)}{[(1-s) \gamma]^{N} \xi_{N}}=\kappa \frac{2 s \varepsilon \xi_{N-1}}{(1-s) \gamma \xi_{N}}\left(1+o_{K}(1)\right)
$$

hence

$$
\sup _{\varepsilon \leqslant \gamma} \frac{\rho(f, \varepsilon, \gamma)}{\varepsilon^{\alpha}} \leqslant \kappa \frac{2 s}{1-s} \cdot \frac{\xi_{N-1}}{\xi_{N}} \cdot \gamma^{-\alpha}\left(1+o_{K}(1)\right)
$$

with

$$
o_{K}(1) \rightarrow 0 \text { as } \gamma \rightarrow 0^{+}
$$

so (PE5) holds.

Proof of Proposition 3.1. We conclude that one can apply Theorem 4.2 for maps in $f \in \mathcal{M}^{*}\left(s, K, \kappa, \varepsilon_{0}\right)$ and $\varepsilon_{L Y}=\varepsilon_{*} \leqslant \varepsilon_{0}$. Bounds for $c_{\operatorname{det} H}, \rho\left(f, \cdot, \varepsilon_{*}\right)$ and $\eta\left(f, \varepsilon_{*}\right)$ are determined by the dimension $N$ and $s, K, \kappa$, using (4.4), (4.5) and (4.6). We obtain

$$
\begin{aligned}
& \gamma_{L Y} \leqslant\left(1+c_{\operatorname{detH}} s^{\alpha} \varepsilon_{L Y}^{\alpha}\right)\left(s^{\alpha}+2 \kappa \frac{2 s \xi_{N-1}}{(1-s) \xi_{N}}\left(1+o_{K}(1)\right)\right. \\
& K_{L Y} \leqslant 2 c_{\operatorname{detH}} s^{\alpha}+4 \kappa\left(1+c_{\operatorname{detH}} s^{\alpha} \varepsilon_{L Y}^{\alpha}\right) \frac{s \xi_{N-1}}{(1-s) \xi_{N}} \varepsilon_{L Y}^{-\alpha}\left(1+o_{K}(1)\right)
\end{aligned}
$$

with

$$
o_{K}(1) \rightarrow 0 \text { as } \varepsilon_{L Y} \rightarrow 0^{+} .
$$

Then, given (2.2) and taking into account the bound (4.4) for $c_{\operatorname{detH}}$, there exists $\varepsilon_{L Y}$ sufficiently small, determined only by the dimension $N$ and $s, K, \kappa, \varepsilon_{0}$, such that $\gamma_{L Y}<1$.

Proof of Lemma 4.3. We only sketch the idea, for more details see [40, Lemma 2.1]. Through a chart, one can map locally $\Gamma$ into a hyperplane of $\mathbb{R}^{N}$. Up to a small distortion, we have now to intersect a $\nu$-neighborhood of the hyperplane by a sphere of radius $\omega$. The largest volume occurs when the center of the sphere is on the hyperplane, and then the intersection is close to a cylinder of height $2 \nu$ and radius $\omega$.

4.2. Preliminaries to the Proof of Theorem 4.2. We begin by showing that $\mathrm{OSC}_{\alpha}$ continuously injects into $L^{\infty}(\mu)$.

Lemma 4.4. Let $\varphi \in L^{1}(\mu)$. If $a, b, c>0$ satisfy $a+b \leqslant c \leqslant 1 / 2$, then for all $x \in \mathbb{T}^{N}$, we have

$$
\operatorname{Esup}(\varphi, B(x, a)) \leqslant \frac{1}{\mu(B(x, b))} \int_{B(x, b)}[\varphi(y)+\operatorname{osc}(\varphi, B(y, c))] d y
$$


Proof of Lemma 4.4. Let $x \in \mathbb{T}^{N}$. For $y \in B(x, b)$, we have $B(x, a) \subset B(y, c)$. Consequently, we have

$$
\operatorname{Esup}(\varphi, B(x, a)) \leqslant \operatorname{Esup}(\varphi, B(y, c)) \leqslant \varphi(y)+\operatorname{osc}(\varphi, B(y, c))
$$

$\mu$ almost everywhere. Now average.

Lemma 4.5 $\left(\mathrm{OSC}_{\alpha}\right.$ continuously injects into $\left.L^{\infty}(\mu)\right)$. For all $\varphi \in \mathrm{OSC}_{\alpha}$, we have

$$
\|\varphi\|_{L^{\infty}(\mu)} \leqslant \frac{\max \left\{1, \varepsilon_{*}^{\alpha}\right\}}{\xi_{N} \varepsilon_{*}{ }^{N}}\|\varphi\|_{\alpha, \varepsilon_{*}} .
$$

Proof of Lemma 4.5. Using Lemma 4.4 with $a>0$ and $b=\varepsilon_{*}-a$ gives

$$
\|\varphi\|_{L^{\infty}(\mu)} \leqslant \frac{\max \left\{1, \varepsilon_{*}^{\alpha}\right\}}{\xi_{N}\left(\varepsilon_{*}-a\right)^{N}}\|\varphi\|_{\alpha, \varepsilon_{*}} .
$$

Now let $a \rightarrow 0$.

Proposition 4.6 (Properties of osc). Let $\varphi,\left(\varphi_{i}\right)_{i=1}^{M}$, and $\psi$ be elements of $L^{\infty}(\mu)$ such that $\psi \geqslant 0$. Let $S$ be a Borel subset of $\mathbb{T}^{N}$.

(O1)

$$
\operatorname{osc}\left(\sum_{i=1}^{M} \varphi_{i}, S\right) \leqslant \sum_{i=1}^{M} \operatorname{osc}\left(\varphi_{i}, S\right) .
$$

(O2) For all $a>0$ and $x \in \mathbb{T}^{N}$ we have

$$
\begin{aligned}
\operatorname{osc}\left(\varphi \mathbf{1}_{S}, B(x, a)\right) \leqslant & \operatorname{osc}(\varphi, S \cap B(x, a)) \cdot \mathbf{1}_{S \backslash B_{a}(\partial S)}(x) \\
& +2 \cdot \operatorname{Esup}(|\varphi|, B(x, a) \cap S) \cdot \mathbf{1}_{B_{a}(\partial S)}(x) \\
\leqslant & \operatorname{osc}(\varphi, S \cap B(x, a)) \cdot \mathbf{1}_{S}(x) \\
& +2 \cdot \operatorname{Esup}(|\varphi|, B(x, a) \cap S) \cdot \mathbf{1}_{B_{a}(\partial S)}(x) .
\end{aligned}
$$

(O3) $\operatorname{osc}(\varphi \psi, S) \leqslant \operatorname{osc}(\varphi, S) \operatorname{Esup}(\psi, S)+\operatorname{osc}(\psi, S) \operatorname{Einf}(|\varphi|, S)$.

Proof of Proposition 4.6. (O1) is immediate.

For $(\mathrm{O} 2)$, we check the first inequality (the second only gives a more convenient expression). It is easy to see that it holds whether $x \in S \backslash B_{a}(\partial S), x \in B_{a}(\partial S)$ or $x$ is in none of these two sets. For the second case use that $\operatorname{osc}(\varphi, S) \leqslant 2 \operatorname{Esup}(|\varphi|, S)$.

For (O3), if $\varphi$ is non-negative $\mu$ almost everywhere, then

$$
\begin{aligned}
\operatorname{osc}(\varphi \psi, S) \leqslant & \operatorname{Esup}(\varphi, S) \operatorname{Esup}(\psi, S)-\operatorname{Einf}(\varphi, S) \operatorname{Einf}(\psi, S) \\
= & \operatorname{Esup}(\psi, S)(\operatorname{Esup}(\varphi, S)-\operatorname{Einf}(\varphi, S)) \\
& +\operatorname{Einf}(\varphi, S)(\operatorname{Esup}(\psi, S)-\operatorname{Einf}(\psi, S)) \\
= & \operatorname{Esup}(\psi, S) \operatorname{osc}(\varphi, S)+\operatorname{Einf}(\varphi, S) \operatorname{osc}(\psi, S)
\end{aligned}
$$

If $\varphi$ is non-positive $\mu$ almost everywhere, we argue similarly using $\operatorname{osc}(\varphi \psi, S)=$ $\operatorname{osc}(-\varphi \psi, S)$. Otherwise, we have

$$
\begin{aligned}
\operatorname{Esup}(\varphi \psi, S)-\operatorname{Einf}(\varphi \psi, S) & =\operatorname{Esup}(\varphi \psi, S)+\operatorname{Esup}(-\varphi \psi, S) \\
& \leqslant \operatorname{Esup}(\psi, S)(\operatorname{Esup}(\varphi, S)+\operatorname{Esup}(-\varphi, S)) \\
& =\operatorname{Esup}(\psi, S) \operatorname{osc}(\varphi, S),
\end{aligned}
$$

so (O3) is proven. 
4.3. Proof of Theorem 4.2 (Lasota-Yorke inequality). Recall that in our setting

$$
\mathcal{P}_{f}(\varphi)=\sum_{i} \frac{\varphi}{|\operatorname{det} D f|} \circ\left(\left.f\right|_{U_{i}}\right)^{-1} \cdot \mathbf{1}_{f\left(U_{i}\right)} \quad \text { a.e. }
$$

Assume $f$ satisfies (PE1)-(PE5), $\varphi \in \mathrm{OSC}_{\alpha}$, and $\varepsilon \leqslant \varepsilon_{*}$. Using (O1) and (O2), for $\mu$ almost every $x \in \mathbb{T}^{N}$, we have

$$
\begin{aligned}
\operatorname{osc}\left(\mathcal{P}_{f} \varphi, B(x, \varepsilon)\right) \leqslant & \sum_{i=1}^{M} \operatorname{osc}\left(\left(\frac{\varphi}{|\operatorname{det}(D f)|} \circ\left(\left.f\right|_{U_{i}}\right)^{-1}\right) \mathbf{1}_{f\left(U_{i}\right)}, B(x, \varepsilon)\right) \\
\leqslant & \sum_{i=1}^{M} \operatorname{osc}\left(\frac{\varphi}{|\operatorname{det}(D f)|} \circ\left(\left.f\right|_{U_{i}}\right)^{-1}, f\left(U_{i}\right) \cap B(x, \varepsilon)\right) \mathbf{1}_{f\left(U_{i}\right)}(x) \\
& +2\left(\operatorname{Esup}\left(\left|\frac{\varphi}{|\operatorname{det}(D f)|} \circ\left(\left.f\right|_{U_{i}}\right)^{-1}\right|, f\left(U_{i}\right) \cap B(x, \varepsilon)\right)\right) \\
& \times \mathbf{1}_{B_{\varepsilon}\left(\partial f\left(U_{i}\right)\right)}(x) \\
\leqslant & \sum_{i=1}^{M} \operatorname{osc}\left(\frac{\varphi}{|\operatorname{det}(D f)|}, U_{i} \cap\left(\left.f\right|_{U_{i}}\right)^{-1}(B(x, \varepsilon))\right) \mathbf{1}_{f\left(U_{i}\right)}(x) \\
& +2\left(\operatorname{Esup}\left(\frac{|\varphi|}{|\operatorname{det}(D f)|}, U_{i} \cap\left(\left.f\right|_{U_{i}}\right)^{-1}(B(x, \varepsilon))\right)\right) \\
& \times \mathbf{1}_{B_{\varepsilon}\left(\partial f\left(U_{i}\right)\right)}(x) .
\end{aligned}
$$

We will check that the $L^{1}$ norm of the right-hand side of (4.9) is bounded by

$$
\gamma_{S}|\varphi|_{\alpha, \varepsilon_{*}} \varepsilon^{\alpha}+K_{S}\|\varphi\|_{L^{1}(\mu)} \varepsilon^{\alpha}
$$

with $\gamma_{S}$ and $K_{S}$ as stated in (4.2) and (4.3).

We estimate the two components of the right-hand side of (4.9) separately. For the first component, define

$$
R_{i}^{(1)}(x):=\operatorname{osc}\left(\varphi|\operatorname{det}(D f)|^{-1}, \quad U_{i} \cap\left(\left.f\right|_{U_{i}}\right)^{-1}(B(x, \varepsilon))\right) .
$$

For $x \in f\left(U_{i}\right)$, setting $z_{i}:=\left(\left.f\right|_{U_{i}}\right)^{-1}(x)$ and using (PE4), we have

$$
R_{i}^{(1)}(x) \leqslant \operatorname{osc}\left(\varphi|\operatorname{det}(D f)|^{-1}, \quad U_{i} \cap B\left(z_{i}, s \varepsilon\right)\right)
$$

(O3) implies that for $\mu$ almost every $x \in \mathbb{T}^{N}$ we have ${ }^{13}$

$$
\begin{aligned}
R_{i}^{(1)}(x) \leqslant & \operatorname{osc}\left(\varphi, U_{i} \cap B\left(z_{i}, s \varepsilon\right)\right) \operatorname{Esup}\left(|\operatorname{det}(D f)|^{-1}, U_{i} \cap B\left(z_{i}, s \varepsilon\right)\right) \\
& +\operatorname{osc}\left(|\operatorname{det}(D f)|^{-1}, U_{i} \cap B\left(z_{i}, s \varepsilon\right)\right) \operatorname{Einf}\left(|\varphi|, U_{i} \cap B\left(z_{i}, s \varepsilon\right)\right) \\
\leqslant & \left(1+c_{\operatorname{detH}}(s \varepsilon)^{\alpha}\right)\left|\operatorname{det}\left(D f\left(z_{i}\right)\right)\right|^{-1} \operatorname{osc}\left(\varphi, U_{i} \cap B\left(z_{i}, s \varepsilon\right)\right) \\
& +2 c_{\operatorname{detH}}(s \varepsilon)^{\alpha}\left|\varphi\left(z_{i}\right)\right| \cdot\left|\operatorname{det}\left(D f\left(z_{i}\right)\right)\right|^{-1} .
\end{aligned}
$$

For Lebesgue almost every $x \in \mathbb{T}^{N}$ the first component of the right-hand side of (4.9) therefore satisfies

$$
\sum_{i=1}^{M} R_{i}^{(1)} \mathbf{1}_{f\left(U_{i}\right)}(x) \leqslant\left(1+c_{\operatorname{detH}}(s \varepsilon)^{\alpha}\right) \mathcal{P}_{f}(\operatorname{osc}(\varphi, B(\cdot, s \varepsilon)))+2 c_{\operatorname{detH}}(s \varepsilon)^{\alpha} \mathcal{P}_{f}|\varphi| .
$$

\footnotetext{
${ }^{13}$ We use that $\operatorname{Einf}\left(|\varphi|, U_{i} \cap B\left(z_{i}, s \varepsilon\right)\right) \leqslant\left|\varphi\left(z_{i}\right)\right|$ for a.e. $x=f\left(z_{i}\right), z_{i} \in U_{i}$.
} 
Integrating over $\mathbb{T}^{N}$ and using (2.5) yields

$$
\int_{\mathbb{T}^{N}} \sum_{i=1}^{M} R_{i}^{(1)} \mathbf{1}_{f\left(U_{i}\right)} d \mu \leqslant\left(1+c_{\operatorname{detH}}(s \varepsilon)^{\alpha}\right)|\varphi|_{\alpha, \varepsilon_{*}}(s \varepsilon)^{\alpha}+2 c_{\operatorname{detH}}(s \varepsilon)^{\alpha}\|\varphi\|_{L^{1}(\mu)} .
$$

For the second component of the right-hand side of (4.9), we use the extensions $f_{(i)}$ to $V_{i}$. Define

$$
R_{i}^{(2)}(x)=\operatorname{Esup}\left(\frac{|\varphi|}{|\operatorname{det}(D f)|}, U_{i} \cap\left(\left.f\right|_{U_{i}}\right)^{-1}(B(x, \varepsilon))\right) \cdot \mathbf{1}_{B_{\varepsilon}\left(\partial f\left(U_{i}\right)\right)}(x) .
$$

and $\operatorname{let}^{14} z_{i}:=f_{(i)}^{-1}(x) \in V_{i}$ for $x \in B_{\varepsilon}\left(\partial f\left(U_{i}\right)\right)$. Using the regularity of $\operatorname{det}\left(D f_{(i)}\right)$, we have

$$
R_{i}^{(2)}(x) \leqslant\left(1+c_{\operatorname{detH}}(s \varepsilon)^{\alpha}\right) \operatorname{Esup}\left(|\varphi|, B\left(z_{i}, s \varepsilon\right)\right)\left|\operatorname{det}\left(D f_{(i)}\left(z_{i}\right)\right)\right|^{-1} \cdot \mathbf{1}_{B_{\varepsilon}\left(\partial f\left(U_{i}\right)\right)}(x) .
$$

Integrating, changing variables $z_{i}=f_{(i)}^{-1}(x)$ and using Lemma 4.4 with $a=s \varepsilon, b=$ $(1-s) \varepsilon_{*}$, and $c=\varepsilon_{*}$ yields

$$
\begin{aligned}
(1+ & \left.c_{\operatorname{detH}}(s \varepsilon)^{\alpha}\right)^{-1} \int_{\mathbb{T}^{N}} R_{i}^{(2)}(x) d \mu(x) \\
\leqslant & \int_{\mathbb{T}^{N}} \operatorname{Esup}\left(|\varphi|, B\left(z_{i}, s \varepsilon\right)\right)\left|\operatorname{det}\left(D f_{(i)}\left(z_{i}\right)\right)\right|^{-1} \cdot \mathbf{1}_{B_{\varepsilon}\left(\partial f\left(U_{i}\right)\right.}(x) d \mu(x) \\
= & \int_{V_{i}} \operatorname{Esup}\left(|\varphi|, B\left(z_{i}, s \varepsilon\right)\right) \cdot \mathbf{1}_{B_{\varepsilon}\left(\partial f\left(U_{i}\right)\right)}\left(f_{(i)}\left(z_{i}\right)\right) d \mu\left(z_{i}\right) \\
\leqslant & \int_{V_{i}} \mathbf{1}_{B_{\varepsilon}\left(\partial f\left(U_{i}\right)\right.}\left(f_{(i)}(z)\right) \\
& \times\left[\frac{1}{\mu\left(B\left(z,(1-s) \varepsilon_{*}\right)\right)} \int_{B\left(z,(1-s) \varepsilon_{*}\right)}\left[|\varphi(\zeta)|+\operatorname{osc}\left(|\varphi|, B\left(\zeta, \varepsilon_{*}\right)\right)\right] d \mu(\zeta)\right] d \mu(z) \\
= & \int_{\mathbb{T}^{N}}\left[|\varphi(\zeta)|+\operatorname{osc}\left(|\varphi|, B\left(\zeta, \varepsilon_{*}\right)\right)\right] \\
& \times\left[\int_{V_{i}} \frac{\mathbf{1}_{f_{(i)}^{-1}\left(B_{\varepsilon}\left(\partial f\left(U_{i}\right)\right)\right)}(z) \cdot \mathbf{1}_{B\left(\zeta,(1-s) \varepsilon_{*}\right)}(z)}{\mu\left(B\left(z,(1-s) \varepsilon_{*}\right)\right)} d \mu(z)\right] d \mu(\zeta) \\
= & \int_{\mathbb{T}^{N}}\left[|\varphi(\zeta)|+\operatorname{osc}\left(|\varphi|, B\left(\zeta, \varepsilon_{*}\right)\right)\right] \\
& \times \frac{\mu\left(f_{(i)}^{-1}\left(B_{\varepsilon}\left(\partial f\left(U_{i}\right)\right)\right) \cap B\left(\zeta,(1-s) \varepsilon_{*}\right)\right)}{\mu\left(B\left(\zeta,(1-s) \varepsilon_{*}\right)\right)} d \mu(\zeta) .
\end{aligned}
$$

We arrive at the estimate

$$
\left(1+c_{\operatorname{detH}}(s \varepsilon)^{\alpha}\right)^{-1} \int_{\mathbb{T}^{N}} \sum_{i=1}^{M} R_{i}^{(2)}(x) d \mu(x) \leqslant \rho\left(f, \varepsilon, \varepsilon_{*}\right)\left(\|\varphi\|_{L^{1}(\mu)}+|\varphi|_{\alpha, \varepsilon_{*}} \varepsilon_{*}{ }^{\alpha}\right) .
$$

\footnotetext{
${ }^{14}$ Here, and in the subsequent change of variables, we use that the extension $f_{(i)}$ is one-to-one near the boundary of $U_{i}$. If this were finite-to-one, we would have to multiply — among others $\eta\left(f, \varepsilon_{*}\right)$ by this multiplicity.
} 
Combining estimates (4.11) and (4.12), we have

$$
\begin{aligned}
\int_{\mathbb{T}^{N}} \operatorname{osc}\left(\mathcal{P}_{f} \varphi, B(x, \varepsilon)\right) d \mu(x) \leqslant & \left(1+c_{\operatorname{detH}} s^{\alpha} \varepsilon^{\alpha}\right)|\varphi|_{\alpha, \varepsilon_{*}} s^{\alpha} \varepsilon^{\alpha}+2 c_{\operatorname{detH}} s^{\alpha} \varepsilon^{\alpha}\|\varphi\|_{L^{1}(\mu)} \\
& +2\left(1+c_{\operatorname{detH}} s^{\alpha} \varepsilon^{\alpha}\right) \rho\left(f, \varepsilon, \varepsilon_{*}\right) \\
& \times\left(\|\varphi\|_{L^{1}(\mu)}+|\varphi|_{\alpha, \varepsilon_{*}} \varepsilon_{*}^{\alpha}\right) .
\end{aligned}
$$

Then inequality (4.10) holds with

$$
\begin{aligned}
\gamma_{S} & =\left(1+c_{\mathrm{detH}} s^{\alpha} \varepsilon_{*}{ }^{\alpha}\right)\left(s^{\alpha}+2 \cdot \sup _{\varepsilon \leqslant \varepsilon_{*}} \frac{\rho\left(f, \varepsilon, \varepsilon_{*}\right)}{\varepsilon^{\alpha}} \varepsilon_{*}{ }^{\alpha}\right)=\left(1+c_{\operatorname{detH}} s^{\alpha} \varepsilon_{*}{ }^{\alpha}\right) \eta\left(f, \varepsilon_{*}\right) \\
K_{S} & =2 c_{\operatorname{detH}} s^{\alpha}+2\left(1+c_{\operatorname{detH}} s^{\alpha} \varepsilon_{*}{ }^{\alpha}\right)\left(\sup _{\varepsilon \leqslant \varepsilon_{*}} \frac{\rho\left(f, \varepsilon, \varepsilon_{*}\right)}{\varepsilon^{\alpha}}\right)
\end{aligned}
$$

as claimed.

\section{References}

[1] K. Adl-Zarabi, Absolutely continuous invariant measures for piecewise expanding $C^{2}$ transformations in $\mathbf{R}^{n}$ on domains with cusps on the boundaries, Ergodic Theory Dynam. Syst. 16(1) (1996), 1-18.

[2] L. Arnold, Random dynamical systems, Springer Monographs in Mathematics, Springer-Verlag, Berlin, 1998, ISBN 3-540-63758-3.

[3] P.H. Baxendale, Stability and equilibrium properties of stochastic flows of diffeomorphisms, in 'Diffusion processes and related problems in analysis', vol. II (Charlotte, NC, 1990), Progr. Probab., 27, 3-35, Birkhäuser Boston, Boston, MA, 1992.

[4] G. Birkhoff, Lattice theory, American Mathematical Society Colloquium Publications 25, American Mathematical Society, Providence, R.I., 3rd ed., 1979, ISBN 0-8218-1025-1.

[5] M.L. Blank, Stochastic properties of deterministic dynamical systems, Math. Phys. Rev., 6, Vol. 6 of Soviet Sci. Rev. Sect. C Math. Phys. Rev., 243-271; Harwood Academic Publ., Chur, 1987.

[6] X. Bressaud and C. Liverani, Anosov diffeomorphisms and coupling, Ergodic Theory Dyn. Syst. 22(1) (2002), 129-152.

[7] J. Buzzi, Absolutely continuous invariant measures for generic multi-dimensional piecewise affine expanding maps, Inter. J. Bifur. Chaos Appl. Sci. Eng. 9(9) (1999), 1743-1750, Discrete dynamical systems.

[8] - Absolutely continuous invariant probability measures for arbitrary expanding piecewise R-analytic mappings of the plane, Ergodic Theory Dyn. Syst. 20(3) (2000), 697-708.

[9] - No or infinitely many a.c.i.p. for piecewise expanding $C^{r}$ maps in higher dimensions, Comm. Math. Phys. 222(3) (2001), 495-501.

[10] J. Buzzi and V. Maume-Deschamps, Decay of correlations for piecewise invertible maps in higher dimensions, Israel J. Math. 131 (2002), 203-220.

[11] N. Chernov, Advanced statistical properties of dispersing billiards, J. Stat. Phys. 122(6) (2006), 1061-1094.

[12] N. Chernov and D. Dolgopyat, Brownian Brownian motion. I, Mem. Amer. Math. Soc. 198(927) (2009), viii+193.

[13] W.J. Cowieson, Absolutely continuous invariant measures for most piecewise smooth expanding maps, Ergodic Theory Dyn. Syst. 22(4) (2002), 1061-1078.

[14] M.F. Demers and C. Liverani, Stability of statistical properties in two-dimensional piecewise hyperbolic maps, Trans. Amer. Math. Soc. 360(9) (2008), 4777-4814.

[15] D. Dolgopyat, Prevalence of rapid mixing in hyperbolic flows, Ergodic Theory Dyn. Syst. 18(5) (1998), 1097-1114.

[16] - Prevalence of rapid mixing. II. Topological prevalence, Ergodic Theory Dyn. Syst. 20(4) (2000), 1045-1059. 
[17] M. Field, I. Melbourne and A. Török, Decay of correlations, central limit theorems and approximation by Brownian motion for compact Lie group extensions, Ergodic Theory Dyn. Syst. 23(1) (2003), 87-110.

[18] P. Góra and A. Boyarsky, Absolutely continuous invariant measures for piecewise expanding $C^{2}$ transformation in $\mathbf{R}^{N}$, Israel J. Math. 67(3) (1989), 272-286.

[19] S. Gouëzel and C. Liverani, Banach spaces adapted to Anosov systems, Ergodic Theory Dyn. Syst. 26(1) (2006), 189-217.

[20] G. Keller, Generalized bounded variation and applications to piecewise monotonic transformations, Z. Wahrsch. Verw. Gebiete 69(3) (1985), 461-478.

[21] H. Kunita, Stochastic flows and stochastic differential equations, Vol. 24 of Cambridge Studies in Advanced Mathematics, Cambridge University Press, Cambridge (1990), ISBN 0-521-35050-6.

[22] A. Lasota and J.A. Yorke, When the long-time behavior is independent of the initial density, SIAM J. Math. Anal. 27(1) (1996), 221-240.

[23] Y. Le Jan, On isotropic Brownian motions, Z. Wahrsch. Verw. Gebiete 70(4) (1985), 609-620.

[24] F. Ledrappier and L.-S. Young, Entropy formula for random transformations, Probab. Theory Related Fields 80(2) (1988), 217-240.

[25] K.K. Lin, E. Shea-Brown and L.-S. Young, Reliability of coupled oscillators, J. Nonlinear Sci. 19(5) (2009), 497-545.

[26] C. Liverani, Decay of correlations, Ann. of Math. (2) 142(2) (1995), 239-301.

[27] - Decay of correlations for piecewise expanding maps, J. Stat. Phys. 78(3-4) (1995), 1111-1129.

[28] C. Liverani, B. Saussol and S. Vaienti, Conformal measure and decay of correlation for covering weighted systems, Ergodic Theory Dyn. Syst. 18(6) (1998), 1399-1420.

[29] N. Masmoudi and L.-S. Young, Ergodic theory of infinite dimensional systems with applications to dissipative parabolic PDEs, Comm. Math. Phys. 227(3) (2002), 461-481.

[30] J.C. Mattingly, Ergodicity of $2 D$ Navier-Stokes equations with random forcing and large viscosity, Commun. Math. Phys. 206(2) (1999), 273-288.

[31] I. Melbourne, Rapid decay of correlations for nonuniformly hyperbolic flows, Trans. Amer. Math. Soc. 359(5) (2007), 2421-2441 (electronic).

[32] - Decay of correlations for slowly mixing flows, Proc. Lond. Math. Soc. (3) 98(1) (2009), $163-190$.

[33] A. Mohapatra and W. Ott, Memory loss for nonequilibrium open dynamical systems, 2013. To appear in Discrete and Continuous Dynamical Systems-Series A.

[34] R. Murray, Existence, mixing and approximation of invariant densities for expanding maps on $\mathbb{R}^{r}$, Nonlinear Anal. A, Theory Methods 45(1) (2001), 37-72.

[35] R.D. Nussbaum, Hilbert's projective metric and iterated nonlinear maps, Mem. Amer. Math. Soc. 75(391) (1988), iv+137.

[36] - Iterated nonlinear maps and Hilbert's projective metric. II, Mem. Amer. Math. Soc. 79(401) (1989), iv+118.

[37] W. Ott, M. Stenlund and L.-S. Young, Memory loss for time-dependent dynamical systems, Math. Res. Lett. 16(3) (2009), 463-475.

[38] H. Proppe, P. Góra and A. Boyarsky, Inadequacy of the bounded variation technique in the ergodic theory of higher-dimensional transformations, Nonlinearity 3(4) (1990), 1081-1087.

[39] O. Sarig, Subexponential decay of correlations, Invent. Math. 150(3) (2002), 629-653.

[40] B. Saussol, Absolutely continuous invariant measures for multidimensional expanding maps, Israel J. Math. 116 (2000), 223-248.

[41] M. Stenlund, L.-S. Young and H. Zhang, Dispersing billiards with moving scatterers. Commun. Math. Phys., (2012), DOI: 10.1007/s00220-013-1746-6

[42] M. Tsujii, Absolutely continuous invariant measures for piecewise real-analytic expanding maps on the plane, Commun. Math. Phys. 208(3) (2000), 605-622.

[43] - Piecewise expanding maps on the plane with singular ergodic properties, Ergodic Theory Dyn. Syst. 20(6) (2000), 1851-1857.

[44] - Absolutely continuous invariant measures for expanding piecewise linear maps, Invent. Math. 143(2) (2001), 349-373. 
[45] L.-S. Young, Statistical properties of dynamical systems with some hyperbolicity, Ann. Math. (2) $\mathbf{1 4 7}(3)$ (1998), 585-650.

[46] — Recurrence times and rates of mixing, Israel J. Math. 110 (1999), 153-188.

Department of Mathematics, University of Houston, 651 Philip G. Hoffman Hall, HousTON, TX 77204-3008, USA

E-mail address: chinmaya@math.uh.edu, URL: http://www.math.uh.edu/ chinmaya/

E-mail address: ott@math.uh.edu, URL:http://www.math.uh.edu/ ott/

E-mail address: torok@math.uh.edu, URL: http://www.math.uh.edu/ torok/ 
